\title{
Magnetic Antivortex-Core Reversal by Circular-Rotational Spin Currents
}

\author{
Thomas Kamionka, ${ }^{1, *}$ Michael Martens, ${ }^{1}$ Kang Wei Chou, ${ }^{2}$ Michael Curcic, ${ }^{3}$ André Drews, ${ }^{4}$ Gisela Schütz, ${ }^{3}$ \\ Tolek Tyliszczak, ${ }^{2}$ Hermann Stoll, ${ }^{3}$ Bartel Van Waeyenberge,${ }^{5}$ and Guido Meier ${ }^{1}$ \\ ${ }^{1}$ Institut für Angewandte Physik und Zentrum für Mikrostrukturforschung, Universität Hamburg, 20355 Hamburg, Germany \\ ${ }^{2}$ Advanced Light Source, LBNL, 94720 Berkeley, California, USA \\ ${ }^{3}$ Max-Planck-Institut für Metallforschung, 70569 Stuttgart, Germany \\ ${ }^{4}$ Arbeitsbereich Technische Informatiksysteme, Universität Hamburg, 22527 Hamburg, Germany \\ ${ }^{5}$ Department of Solid State Sciences, Ghent University, 9000 Ghent, Belgium
}

(Received 23 March 2010; published 21 September 2010)

\begin{abstract}
Topological singularities occur as antivortices in ferromagnetic thin-film microstructures. Antivortices behave as two-dimensional oscillators with a gyrotropic eigenmode which can be excited resonantly by spin currents and magnetic fields. We show that the two excitation types couple in an opposing sense of rotation in the case of resonant antivortex excitation with circular-rotational currents. If the sense of rotation of the current coincides with the intrinsic sense of gyration of the antivortex, the coupling to the Oersted fields is suppressed and only the spin-torque contribution locks into the gyrotropic eigenmode. We report on the experimental observation of purely spin-torque induced antivortex-core reversal. The dynamic response of an isolated antivortex is imaged by time-resolved scanning transmission x-ray microscopy on its genuine time and length scale.
\end{abstract}

DOI: 10.1103/PhysRevLett.105.137204

Magnetization dynamics in microstructures is not only an aspect of basic research. Its investigation is also motivated by the vision of engineering new storage devices. Magnetic antivortices and vortices are capable of storing binary information [1-3] represented by their core polarization $p= \pm 1$ and behave as two-dimensional oscillators with a gyrotropic eigenmode which can be excited resonantly by spin currents and magnetic fields [4-7]. With the perspective of possible applications in storage devices, spin-torque induced core switching is preferred because in this case single elements can be addressed, which is obviously important for storage cells of high density. Another motivator to investigate the current induced dynamics of magnetization is the patent for the magnetic race track memory $[8,9]$, which is based on the unidirectional control of magnetic domain walls in a nanowire by spinpolarized currents [10]. Thereto, a detailed understanding of the coupling between the spin of the conduction electrons and the magnetization of ferromagnets, namely, the spin-transfer torque [11-13], has to be gathered. Nevertheless, experiments on spin-torque driven magnetization dynamics come along with a general difficulty: the discrimination between the spin-transfer torque and the torque from parasitic Oersted fields as driving force [4-6,14-16]. Both torques are generated simultaneously by the applied current. In this Letter we show that both excitation types couple in an opposing sense of rotation in the case of resonant antivortex excitation with circular-rotational currents. The magnetic coupling is suppressed and only the spin-torque contribution locks into the gyrotropic eigenmode if the sense of rotation of the applied current coincides with the intrinsic sense of gyration of the antivortex. We report on the first experimental
PACS numbers: 75.60.Jk, 68.37.Yz, 72.25.-b, 75.60.Ch

observation of purely spin-torque induced antivortex-core reversal.

Antivortices and vortices form in ferromagnetic thinfilm structures [17-19] by a compromise between the shape anisotropy, which prefers the magnetization being in-plane of the film as well as aligned parallel to the edges of the structure, and between the short range exchange coupling, which favors a parallel orientation within the material. Both magnetization configurations are topological counterparts with opposite winding numbers [6]. Figure 1 shows an "infinity-shaped" permalloy $\left(\mathrm{Ni}_{80} \mathrm{Fe}_{20}\right)$ structure with suitable dimensions to stabilize an isolated antivortex [20]. When circulating around the core, the inplane magnetization turns opposite to the sense of circulation described by the winding number $n=-1$, and, therefore, the four surrounding domains alternately point toward and away from the core. These domains continue into the loops of the structure to close the magnetic flux.

For small deflections from the equilibrium position, the core dynamics of antivortices and vortices can be described with the model of a weakly damped harmonic oscillator with the restoring potential given by the stray-field energy [4-6]. The model describes a spiral trajectory into the equilibrium position after a core displacement. With respect to the polarization, this damped gyration follows the left-hand grip rule for antivortices and the right-hand grip rule for vortices. For vortices it has been shown that an inplane rotating magnetic field only excites this gyration mode if the sense of rotation of the field coincides with this intrinsic sense of gyration [2,21]. This gives the opportunity of selective core switching via the sense of rotation of the field. We will show that in contrast to vortices a rotating magnetic field can only resonantly lock into the gyration of 




FIG. 1 (color). Sample for antivortex excitation with circularrotational currents. The atomic-force micrograph of the $t=$ $(53 \pm 1) \mathrm{nm}$ thick permalloy structure is overlaid by the in-plane magnetization imaged by scanning transmission $\mathrm{x}$-ray microscopy. Different color codes accentuate the topography of the electrodes and the in-plane magnetization. A corresponding micromagnetic simulation illustrates the magnetization configuration of the experiment. The exciting circular-rotational current is generated by a superposition of alternating currents through the horizontal and vertical electrode pairs [20].

antivortices if the sense of rotation of the field is opposing the intrinsic sense of gyration. Before the resonant excitation is discussed, Fig. 2 illustrates the coupling of antivortices and vortices to a quasistatic rotating field. Note that the following gedanken experiment proves that only the antivortex shows an inverse dynamical coupling to rotating fields. In Fig. 2 the demagnetization field is always compensated by the quasistatic rotating field $\mathbf{H}_{\mathrm{qs}}$ during the depicted sequences which correspond to one circulation around the center. Therefore, the influence of the demagnetization field is equivalent to the influence of a homogeneous rotating field with the same sense of rotation as $\mathbf{H}_{\mathrm{qs}}$, which only counterrotates in case of the antivortex. Both the antivortex and the vortex would relax by spiral trajectories to the center when the external field would abruptly vanish (see above), but only the antivortex shows the inverse coupling to rotating fields during this motion as it is driven by the demagnetization field.

Recent theoretical publications on current driven antivortex and vortex dynamics deal with spin-transfer torque and the influence of accompanying Oersted fields [4-6,20]. Following this discussion, we consider a circular-rotational current $\mathbf{j}=j_{0} e^{i \Omega t}\left(\mathbf{e}_{x}-i \mathbf{e}_{y}\right)$ and a circular-rotational Oersted field $\mathbf{H}=H_{0} e^{i \Omega t}\left(\right.$ ir $\left.\mathbf{e}_{x}+\mathbf{e}_{y}\right)$ of frequency $\Omega$, which is always aligned perpendicularly to the current,

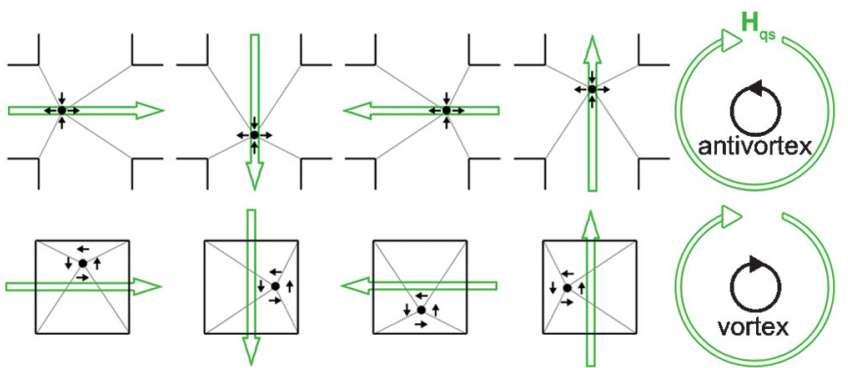

FIG. 2 (color). Coupling of an antivortex (upper sequence) and a vortex (lower sequence) to a quasistatic rotating magnetic field $\mathbf{H}_{\mathrm{qs}}$ represented by green arrows. The Zeeman energy is minimized by the illustrated core deflections because of the increase of the volume of the domain which has a parallel magnetization with respect to the field. Both sequences correspond to one circulation (black circles), whereas $\mathbf{H}_{\mathrm{qs}}$ only counterrotates in the case of the antivortex.

and obtain the equation of motion for antivortices [5]. Both the current and the field have the same sense of rotation $r$, whereas $r=-1$ denotes a clockwise and $r=$ 1 denotes a counterclockwise excitation. We assume low damping and that the degree of nonadiabaticity $\xi$ equals the Gilbert damping parameter $\alpha$ [22]. The equation of motion is solved by

$$
\left(\begin{array}{l}
X \\
Y
\end{array}\right)=-e^{i \Omega t} \chi\left(\begin{array}{r}
i\left[\{\Omega-\omega r p\} v_{j}+\{\Omega p+\omega r\} v_{H}\right] \\
-p\left[\{\omega-\Omega r p\} v_{j}+\{\omega p+\Omega r\} v_{H}\right]
\end{array}\right)
$$

with the chirality of the antivortex [6] $c=0$, which corresponds to the experimental conditions shown in Fig. 1. The factor $\chi=1 /\left[\omega^{2}+(i \Omega+\Gamma)^{2}\right]$ is the susceptibility of the harmonic oscillator model with the eigenfrequency $\omega$ and the damping constant $\Gamma$. The velocities $v_{j}=b_{j} j_{0}$ and $v_{H}=\gamma H_{0} l /(2 \pi)$ describe the coupling of the antivortex core to the adiabatic spin-transfer torque via the gradient of the magnetization and to the magnetic field via the domains. Here, $b_{j}$ is the coupling constant between the current and the magnetization, $\gamma$ is the gyromagnetic ratio, and $l$ is the edge length of the inner square of the "infinity" structure. At resonance $(\Omega=\omega)$, a circular core trajectory is given by

$$
\left(\begin{array}{l}
X \\
Y
\end{array}\right)=\left\{\begin{array}{ll}
-e^{i \omega t} \chi\left(\begin{array}{c}
i \\
-p
\end{array}\right) 2 \omega v_{j} & \text { for } r p=-1 \\
-e^{i \omega t} \chi\left(\begin{array}{c}
i \\
-p
\end{array}\right) 2 \omega p v_{H} & \text { for } r p=1
\end{array} .\right.
$$

Depending on the sense of rotation of the current with respect to the polarization, the core trajectory is determined either by the velocity $v_{j}$ or the velocity $v_{H}$. Thus, only one of the concomitant torques of the circularrotational current drives the antivortex at resonance. We emphasize that the analogous calculation for a vortex does not result in separated resonances for the competing driving forces. In this case both the electron spin and the Oersted field simultaneously couple either constructively or destructively. Because of the inverse reaction of an 
antivortex to rotating magnetic fields, the effect of the spintransfer torque is completely separated from the torque generated by the Oersted field. If the sense of rotation of the current coincides with the intrinsic sense of gyration, the resonant motion is purely driven by spin torque [23]. If the sense of rotation of the current is opposite to the intrinsic sense of gyration, the resonance is purely driven by the Oersted field [24]. Hitherto methods for vortices try to deduce the contribution of the spin-torque effect from partially sensitive or hardly accessible details of the core motion which are related to the ratio between both forces. The analysis of elliptical trajectories for nonresonant linear excitation is problematic due to the breakdown of the gyration amplitude [4]. The measurement of the phase between the gyration and the harmonic excitation is difficult because of signal propagation delays at high frequencies and limited to methods providing both time and spatial resolution [14]. For pulsed excitation [25], the angle of the initial acceleration away from the equilibrium position also depends on the driving mechanism but requires picosecond time resolution and is a sensitive detail of the core response because structural defects of real samples can influence the equilibrium position significantly. All these problems or restrictions vanish from the outset in the case of resonant antivortex excitation with circular-rotational currents as the two possible driving mechanisms couple in the opposite sense of rotation.

For an experimental investigation of antivortex-core switching, we use time-resolved scanning transmission x-ray microscopy [3] at the Advanced Light Source in Berkeley, CA, USA (beam line 11.0.2). The x-ray circular dichroism provides the magnetic contrast as X-ray transmission depends on the projection of the magnetization on the propagation direction of the synchrotron light. To obtain magnetic contrast from the permalloy $\left(\mathrm{Ni}_{80} \mathrm{Fe}_{20}\right)$ layer, a photon energy of $852.7 \mathrm{eV}$ ( $\mathrm{Ni} L_{3}$-absorption edge) is chosen. First the sample is imaged with an angle of $30^{\circ}$ between the normal of the sample plane and the beam axis. This way a strong contrast due to the in-plane magnetization is observed. The domain structure is in excellent agreement with the simulation [see Fig. 1]. All dynamic measurements are performed with a parallel alignment between the normal of the sample plane and the beam axis. Thus, the out-of-plane component of the magnetization is imaged, which is ideal to determine the core polarization and to map the core trajectory [26].

By superposition of alternating currents, the antivortex core is excited with a circular-rotational current [20] of different frequencies in steps of $10 \mathrm{MHz}$. The strongest response is found at $140 \mathrm{MHz}$, which is close to the resonance frequency estimated by micromagnetic simulations [20]. The sense of gyration of this response follows the left-hand grip rule with respect to the core polarization [see Fig. 3] as expected for the antivortex state. This strong resonant gyration of the antivortex core could only be observed with the sense of rotation of the current coinciding with the intrinsic sense of gyration of the antivortex.

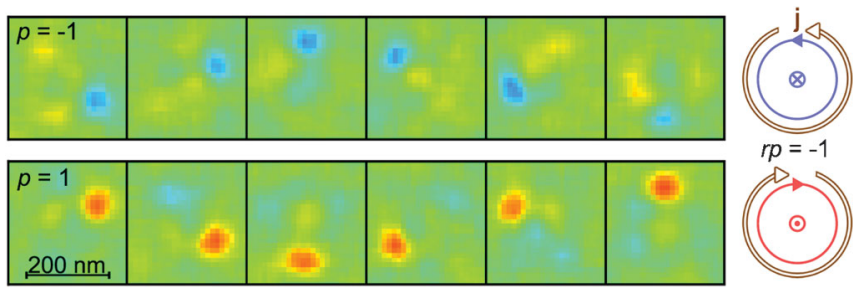

FIG. 3 (color). Resonant gyration of an antivortex with negative (upper sequence) and positive (lower sequence) polarization imaged by time-resolved scanning transmission $\mathrm{x}$-ray microscopy. The intrinsic sense of gyration of the antivortex follows the left-hand grip rule (see blue and red trajectories). As represented by brown arrows, the sense of rotation of the current coincides with the sense of gyration of the core. Because of the inverse coupling, the concomitant Oersted field can not lock into this eigenmode, and consequently the resonant motion of the antivortex is purely driven by the spin-transfer torque. The rotating current has a frequency of $140 \mathrm{MHz}$ and a density of $8.1 \times$ $10^{10} \mathrm{~A} / \mathrm{m}^{2}$. The time step between the images is $1.19 \mathrm{~ns}$. A temporally averaged image is subtracted to eliminate background signals, whereas a core of negative (positive) polarization appears blue (red) due to its negative (positive) out-of-plane components. The actual core size is below the spacial resolution of the microscope [26].

Consequently, the coupling of the spin-transfer torque is much more effective than the coupling of possible Oersted fields within this isolated antivortex. A current density of $8.1 \times 10^{10} \mathrm{~A} / \mathrm{m}^{2}$ causes a gyration amplitude of $(93 \pm$ 5) $\mathrm{nm}$ for the clockwise gyration and $(96 \pm 5) \mathrm{nm}$ for the counterclockwise gyration. The experiment shows that the applied current is nearly able to toggle the core polarization as deformations of the core [27] can be seen in Fig. 3 and in the movies $S 1$ and $S 2$ of the supplementary material [20]. Figure 4 shows a complete cycle of switching the antivortex core $[28,29]$ between both polarization states. Starting with a core polarization of $p=1$ [Fig. 4, lower left panel] and a clockwise rotating current $(r=-1)$, a strong clockwise spin-torque-driven gyration is observed. This situation corresponds to the upper line in Eq. (2). After a slight increase of the current density, the core polarization has been reversed and the dynamic response nearly vanishes [Fig. 4, upper left panel]. This situation corresponds to the lower line in Eq. (2). Note that this unidirectional switching is only possible with the field torque being smaller than the spin-transfer torque. The sense of rotation of the current has not been changed between the lower and the upper left panels in Fig. 4. Thus, after the core reversal only the field torque couples constructively to the new core polarization. However, this coupling is obviously not strong enough to drive a significant gyration. After decreasing the current density back to $8.1 \times 10^{10} \mathrm{~A} / \mathrm{m}^{2}$ and inversing the sense of rotation of the current, the spin-torque driving results again in a strong but counterclockwise gyration [Fig. 4, upper right panel]. To complete the cycle, the core polarization is switched back by increasing the current density above the threshold for 


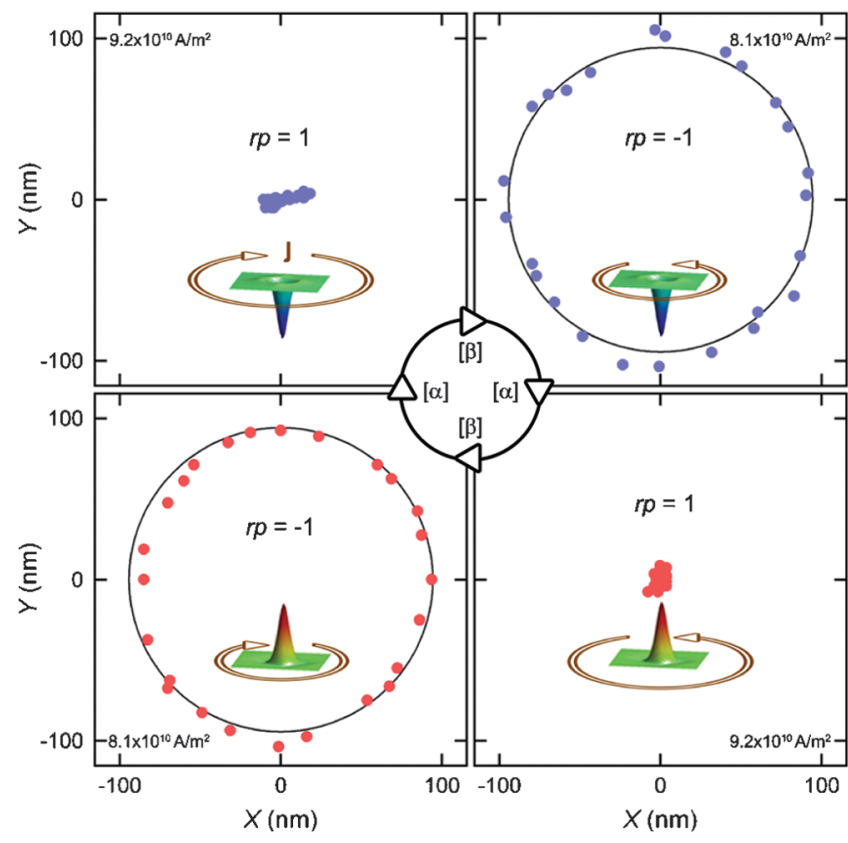

FIG. 4 (color). Experimental cycle of spin-torque induced antivortex-core switching. The sense of rotation and the amplitude of the exciting current is symbolized by the brown arrows within each panel. The blue (red) dots represent experimental results for a negative (positive) core polarization. For a given core polarization, the antivortex shows a strong resonant response (fitted with circular trajectories) with constructive spintorque coupling $(r p=-1)$. An increase of the current density $[\alpha]$ results in an unidirectional switching to the opposite polarization. The core polarization does not toggle back and forth in spite of the high current density but keeps the new polarization with constructive field coupling $(r p=1)$, which only results in a weak response. Before switching back to the starting polarization, the core has to be excited resonantly by the spin-transfer torque again. Therefore, the current density is decreased again and the sense of rotation of the current is reversed $[\beta]$.

core reversal. After the core reversal, we observed again a weak core motion in spite of the high current density [Fig. 4, lower right panel]. Altogether the weak core motions within a radius of $\approx 15 \mathrm{~nm}$ for $r p=1$ (at a $13 \%$ higher current density) compared with the high gyration amplitudes of $(93 \pm 5) \mathrm{nm}$ and $(96 \pm 5) \mathrm{nm}$ for $r p=-1$ show that the field torque is an order of magnitude less effective than the spin-transfer torque. The time-resolved scanning transmission $\mathrm{x}$-ray micrographs which delivered the trajectories shown in Fig. 4 are integrated in the supplementary information as one joint movie $S 3$ [20].

To summarize, the equation of motion for antivortices has been solved for a rotating spin current and a rotating magnetic field of the same sense of rotation. The resonant antivortex-core motion is either caused by the spin-transfer torque or the magnetic torque depending on the sense of rotation. By analyzing the switching scheme of an antivortex core within this model, we experimentally demonstrated antivortex-core reversal by spin-transfer torque. Therefore, not only vortices but also antivortices could be used in nonvolatile data storage devices. For future experiments, the fundamental separation of both torques drastically simplifies the investigation of the interaction between the conduction electrons and the local magnetization and opens further perspectives to study antivortexcore dynamics driven by circular-rotational currents.

We would like to thank René Eiselt for sharing his experience on sample preparation on $\mathrm{Si}_{3} \mathrm{~N}_{4}$ membranes and Ulrich Merkt for continuous support. Financial support by the Deutsche Forschungsgemeinschaft via the Sonderforschungsbereich 668 and the city of Hamburg via the Landesexzellenzcluster Nano-Spintronics is gratefully acknowledged. The Advanced Light Source is supported by the Director, Office of Science, Office of Basic Energy Sciences of the U.S. Department of Energy.

*tkamionk@physnet.uni-hamburg.de

[1] A. Drews et al., Appl. Phys. Lett. 94, 062504 (2009).

[2] S. K. Kim et al., Appl. Phys. Lett. 92, 022509 (2008).

[3] B. Van Waeyenberge et al., Nature (London) 444, 461 (2006).

[4] B. Krüger et al., Phys. Rev. B 76, 224426 (2007).

[5] B. Krüger et al., J. Appl. Phys. 103, 07 A501 (2008).

[6] A. Drews et al., Phys. Rev. B 77, 094413 (2008).

[7] K. Y. Guslienko et al., J. Appl. Phys. 91, 8037 (2002).

[8] S. S. P. Parkin, U.S. Patent No. 3096834005 (2004).

[9] S. S. P. Parkin et al., Science 320, 190 (2008).

[10] M. Hayashi et al., Science 320, 209 (2008).

[11] L. Berger, Phys. Rev. B 54, 9353 (1996).

[12] J. C. Slonczewski, J. Magn. Magn. Mater. 159, L1 (1996).

[13] S. Zhang and Z. Li, Phys. Rev. Lett. 93, 127204 (2004).

[14] M. Bolte et al., Phys. Rev. Lett. 100, 176601 (2008).

[15] L. Bocklage et al., Phys. Rev. B 78, 180405(R) (2008).

[16] S. Kasai et al., Phys. Rev. Lett. 101, 237203 (2008).

[17] K. Shigeto et al., Appl. Phys. Lett. 80, 4190 (2002).

[18] K. Kuepper et al., Phys. Rev. Lett. 99, 167202 (2007).

[19] T. Shinjo et al., Science 289, 930 (2000).

[20] See supplementary material at http://link.aps.org/ supplemental/10.1103/PhysRevLett.105.137204 for supplementary information about sample preparation, micromagnetic simulations, circular-rotational currents, parasitic Oersted fields, clover samples, as well as the supplementary movies $S 1, S 2$, and $S 3$.

[21] M. Curcic et al., Phys. Rev. Lett. 101, 197204 (2008).

[22] M. Hayashi et al., Phys. Rev. Lett. 96, 197207 (2006).

[23] With parameters corresponding to the micromagnetic simulations [20], an amplitude of $100 \mathrm{~nm}$ requires a minimum current density of $3.3 \times 10^{10} \mathrm{~A} / \mathrm{m}^{2}$ according to Eq. (2) for a fully spin-polarized current.

[24] With parameters corresponding to the micromagnetic simulations, an amplitude of $100 \mathrm{~nm}$ is already effected by "small" fields of $\approx 73 \mathrm{~A} / \mathrm{m}$ according to Eq. (2). In Ref. [20] the meaning of "small" is explained in detail.

[25] M. Weigand et al., Phys. Rev. Lett. 102, 077201 (2009).

[26] K. W. Chou et al., Appl. Phys. Lett. 90, 202505 (2007).

[27] A. Vansteenkiste et al., Nature Phys. 5, 332 (2009).

[28] S. Gliga et al., Phys. Rev. B 77, 060404(R) (2008).

[29] S. Gliga et al., J. Appl. Phys. 103, 07B115 (2008). 